\title{
Phylogeny and morphology of Helicotubeufia gen. nov., with three new species in Tubeufiaceae from aquatic habitats
}

\author{
Liu JK ${ }^{1,2}$, Lu YZ ${ }^{3,4}$, Cheewangkoon $\mathbf{R}^{1^{*}}$ and To-Anun $\mathrm{C}^{1^{*}}$
}

${ }^{1}$ Department of Entomology and Plant Pathology, Faculty of Agriculture, Chiang Mai University, Chiang Mai 50200, Thailand

${ }^{2}$ Guizhou Key Laboratory of Agricultural Biotechnology, Guizhou Academy of Agricultural Science, Guiyang 550006, Guizhou, P.R. China

${ }^{3}$ Center of Excellence in Fungal Research, Mae Fah Luang University, Chiang Rai, 57100, Thailand

${ }^{4}$ School of Pharmaceutical Engineering, Guizhou Institute of Technology, Guiyang 550003, Guizhou, P.R. China

Liu JK, Lu YZ, Cheewangkoon R, To-Anun C 2018 - Phylogeny and morphology of Helicotubeufia gen. nov., with three new species in Tubeufiaceae from aquatic habitats. Mycosphere 9(3), 495-509, Doi 10.5943/mycosphere/9/3/4

\begin{abstract}
We are participating in an investigation of freshwater fungi along a north-south latitudinal gradient in the Asian region. Five new collections of asexual and sexual morphs of Tubeufiaceae from decaying wood in freshwater streams were obtained from China and Thailand. Morphologically, they line well with Tubeufiaceae in having superficial, solitary, scattered, subglobose to ellipsoidal-ovate, dark brown to black ascomata; cylindrical asci with hyaline, fusiform, multi-septate, slightly curved and guttulate ascospores and hyphomycetous helicosporous asexual morphs. Phylogenetic analyses based on combined LSU, ITS and TEF1 $\alpha$ sequences data indicate that they formed a monotypic clade among the genera of Tubeufiaceae, but as a phylogenetically distinct lineage. Therefore, we introduce a new genus Helicotubeufia gen. nov, with three species (H. guangxiensis, $H$. hydei and $H$. jonesii) to accommodate these new taxa. Detailed descriptions and illustrations are provided, as well as the comparisons with similar taxa.
\end{abstract}

Key words -4 new taxa - asexual morph - Dothideomycetes - freshwater - Taxonomy

\section{Introduction}

Freshwater fungi are a taxonomically highly diverse group that plays an important role in nutrient cycling and ecosystem functioning (Wong et al. 1998, Vijaykrishna et al. 2006, Shearer et al. 2009, Hyde et al 2016). Most freshwater fungi are distributed in the classes Dothideomycetes and Sordariomycetes of the Ascomycota (Vijaykrishna et al. 2006, Shearer et al. 2009, Hyde et al. 2013, Maharachchikumbura et al. 2015). The Tubeufiales (order of Dothideomycetes) is a large order comprising more than 20 genera of freshwater ascomycetes (Brahmanage et al. 2017), which include both asexual and sexual morphs (Wijayawardene et al. 2017, 2018). The order Tubeufiales was introduced by Boonmee et al. (2014) with a modern treatment based on the examinations of the type specimens and phylogenetic analyses. Three families are currently included in this order (Liu et al. 2017, Wijayawardene et al. 2018). Members of Tubeufiaceae are mostly saprobic and widely distributed, and often found on woody substrates in terrestrial and freshwater habitats (Hyde \& Goh 
1998, Ho et al. 2001, Cai et al. 2002, Zhao et al. 2007, Boonmee et al. 2011, 2014, Hyde et al. 2013, Lu et al. 2017a, c) and sometimes in peat swamps (Pinnoi et al. 2006, Pinruan et al. 2007).

We are carrying out a study of lignicolous freshwater fungi along a north-south latitudinal gradient in Asia (Hyde et al. 2016). In this study, fresh collections of tubeufiaceous taxa were obtained from submerged wood in freshwater stream in China and Thailand respectively. Five taxa formed a monotypic clade among the genera of Tubeufiaceae and showed closely phylogenetic relationship with Aquaphila and Chlamydotubeufia. A new genus Helicotubeufia with three species is introduced and detailed descriptions and illustrations are provided for these new taxa. The phylogenetic analysis of combined LSU, ITS and TEF $1 \alpha$ sequences data is performed to justify the establishment of the new genus, as well as the phylogenetic relationships among the genera of Tubeufiaceae.

\section{Materials \& Methods}

\section{Isolation and morphology}

Decaying wood specimens were collected from Guangxi, China and Trat, Thailand. The samples were processed followed by Boonmee et al. (2014). Morphological observations were made using a Motic SMZ 168 Series stereomicroscope and photographed by a Nikon E80i microscope-camera system. Measurements were made with the Tarosoft (R) Image Frame Work (Liu et al. 2010).

Isolations were made from single spores as described in Liu et al. (2010). Type material is deposited at the herbarium of Cryptogams Kunming Institute of Botany Academia Sinica (HKAS), Kunming, Guizhou Academy of Agricultural Sciences (GZAAS), Guiyang, China, and Mae Fah Luang University (MFLU), Chiang Rai, Thailand. The strains isolated in our study are deposited at Mae Fah Luang University Culture Collection (MFLUCC), Chiang Rai, Thailand and Guizhou Culture Collection (GZCC), Guiyang, China. Facesoffungi numbers and Index Fungorum numbers are provided as outlined in Jayasiri et al. (2015) and Index Fungorum (2018).

\section{DNA extraction, PCR amplification and sequencing}

Fungal isolates were grown on PDA for 14 days at $28{ }^{\circ} \mathrm{C}$ in the dark. Genomic DNA was extracted from the fresh mycelium using Biospin Fungus Genomic DNA Extraction Kit (BioFlux ${ }^{\circledR}$ ) following the manufacturer's protocol (Hangzhou, P.R. China).

DNA amplification was performed by Polymerase Chain Reaction (PCR). Two partial gene portions and one protein coding gene were used in this study: the large subunits of the nuclear ribosomal RNA genes (LSU), the internal transcribed spacers (ITS) and the translation elongation factor 1-alpha gene (TEF1 $\alpha$ ). The primers used were LROR and LR5 (Vilgalys \& Hester 1990) for LSU, ITS5 and ITS4 (White et al. 1990) for ITS, EF1-983F and EF1-2218R (Rehner \& Buckley $2005)$ for TEF $1 \alpha$. The PCR thermal cycle program for LSU, ITS and TEF $1 \alpha$ amplification were as follows: initially denaturing step of $94{ }^{\circ} \mathrm{C}$ for $3 \mathrm{~min}$, followed by 35 cycles of denaturation at $94{ }^{\circ} \mathrm{C}$ for 30 seconds, annealing at $55{ }^{\circ} \mathrm{C}$ for 50 seconds, elongation at $72{ }^{\circ} \mathrm{C}$ for $1 \mathrm{~min}$, and final extension at $72{ }^{\circ} \mathrm{C}$ for $10 \mathrm{~min}$.

PCR products were purified using minicolumns, purification resin and buffer according to the manufacturer's protocols (Amersham product code: 27-9602-01). Sequence analysis was carried out by Shanghai Sangon Biological Engineering Technology and Services Co., Ltd (Shanghai, P.R. China).

\section{Phylogenetic analysis}

Sequences generated from different primers were analyzed with other sequences obtained from GenBank. The related sequences were determined by using a BLAST search to reveal the closest matches with taxa in Tubeufiaceae and recent relevant publications (Boonmee et al. 2011, 2014, Brahmanage et al. 2017, Lu et al. 2017a, b, c, 2018, Luo et al. 2017, Phookamsak et al. 2018). Sequences were aligned using Bioedit 7.2.5 (Hall 1999) and ClustalX v. 1.83 (Thompson et 
al. 1997). The alignments were checked visually and improved manually where necessary. Phylogenetic analyses were performed by using PAUP v. $4.0 b 10$ (Swofford 2002) for maximumparsimony (MP) and MrBayes v. 3.0b4 (Huelsenbeck \& Ronquist 2001) for Bayesian analyses.

A maximum likelihood analysis was performed at the CIPRES webportal (Miller et al. 2010) using RAxML v.7.2.8 as part of the "RAxML-HPC2 on TG" tool (Stamatakis 2006). A general time reversible model (GTR) was applied with a discrete gamma distribution and four rate classes. Fifty thorough maximum likelihood (ML) tree searches were done in RAxML v. 7.2.7 under the same model. One thousand non parametric bootstrap iterations were run with the GTR model and a discrete gamma distribution. The resulting replicates were plotted on to the best scoring tree obtained previously.

Maximum-parsimony analyses were performed using the heuristic search option with 1000 random taxa addition and tree bisection and reconnection (TBR) as the branch-swapping algorithm. All characters were unordered and of equal weight and gaps were treated as missing data. Maxtrees were unlimited, branches of zero length were collapsed and all multiple, equally parsimonious trees were saved. Clade stability was assessed using a bootstrap (BT) analysis with 1000 replicates, each with 10 replicates of random stepwise addition of taxa (Hillis \& Bull 1993). The model of evolution was estimated by using MrModeltest 2.2 (Nylander 2004). Posterior probabilities (PP) (Rannala \& Yang 1996, Zhaxybayeva \& Gogarten 2002) were determined by Markov Chain Monte Carlo sampling (BMCMC) in MrBayes v. 3.0b4 (Huelsenbeck \& Ronquist 2001). Four simultaneous Markov chains were run for 10,000,000 generations and trees were sampled every 1000th generation, thus 10,000 trees were obtained. The suitable burn-in phases were determined by inspecting likelihoods and parameters in Tracer version 1.6 (Rambaut et al. 2013). Based on the tracer analysis, the first 1,000 trees representing $10 \%$ were discarded as the burn-in phase in the analysis. The remaining trees were used to calculate posterior probabilities in the majority rule consensus tree (critical value for the topological convergence diagnostic set to 0.01).

Phylogenetic trees were drawn using Treeview (Page 1996) and MEGA5 (Tamura et al. 2011). Sequences derived in this study are deposited in GenBank (Table 1).

\section{Results}

\section{Phylogenetic analysis}

Five isolates of tubeufiaceous taxa obtained from the submerged decaying wood in the freshwater stream were identified in the family Tubeufiaceae. LSU, ITS and TEF1 $\alpha$ sequence data and morphological characters were used to determine their placement and to describe novel taxa with a comparison with similar taxa.

The combined LSU, ITS and TEF1 $\alpha$ data set comprised 49 taxa with Botryosphaeria dothidea (CBS 115476) as the outgroup taxon. The dataset comprises 2,267 positions (LSU: 1-820; ITS: 821-1437; TEF 1 $\alpha$ : 1438-2267) after alignment, including gaps. The maximum parsimonious dataset consists of 2,267 characters, of which 1,431 characters were constant, and 165 variable characters are parsimony-uninformative. Maximum parsimony analysis of the remaining 671 parsimony-informative characters resulted in 1000 trees with $\mathrm{TL}=3697, \mathrm{CI}=0.382, \mathrm{RI}=0.582$, $\mathrm{RC}=0.223, \mathrm{HI}=0.618$. RAxML, Maximum-parsimony (MP) and Bayesian analysis of the combined dataset resulted in phylogenetic reconstructions with largely similar topologies, and the Bayesian tree is shown in Fig. 1.

Representatives of the sequenced genera (with molecular data) of Tubeufiaceae (Tsui \& Berbee 2006, Tsui et al. 2006, 2007, Promputtha \& Miller 2010, Boonmee et al. 2011, 2014, Rajeshkumar \& Sharma 2013, Brahmanage et al. 2017, Doilom et al. 2017, Lu et al. 2017a, b, 2018, Luo et al. 2017, Phookamsak et al. 2018) are included in our phylogenetic analysis (Fig. 1). Twenty genera are represented by at least one species in Tubeufiaceae included the asexual and sexual morphs. The five newly generated isolates formed a well-supported monotypic clade and can be identified as a new genus (namely as Helicotubeufia) in Tubeufiaceae, and three species were recognized in Helicotubeufia. Helicotubeufia is phylogenetically close to Aquaphila and 
Table 1 Isolates used in this study. Newly deposited sequences are shown in bold

\begin{tabular}{|c|c|c|c|c|c|}
\hline \multirow[t]{2}{*}{ Taxon } & \multirow{2}{*}{$\begin{array}{l}\text { Culture Accession } \\
\text { No. }{ }^{1}\end{array}$} & \multicolumn{3}{|c|}{ GenBank Accession No. ${ }^{2}$} & \multirow[t]{2}{*}{ References } \\
\hline & & ITS & $\overline{\text { LSU }}$ & 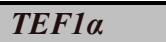 & \\
\hline Acanthohelicospora aurea & GZCC $16-0059^{\mathrm{T}}$ & KY321322 & KY321325 & KY792599 & Lu et al. (2017a) \\
\hline Acanthohelicospora pinicola & MFLUCC $10-0116^{\mathrm{T}}$ & KF301526 & KF301534 & KF301555 & Boonmee et al. (2014) \\
\hline Acanthostigma chiangmaiensis & MFLUCC $10-0125^{\mathrm{T}}$ & JN865209 & JN865197 & KF301560 & Boonmee et al. (2014) \\
\hline Acanthostigma perpusillum & UAMH 7237 & AY916492 & AY856892 & a & Tsui and Berbee (2006) \\
\hline Aquaphila albicans & BCC 3543 & DQ341096 & DQ341101 & & Tsui et al. (2007) \\
\hline Aquaphila asiana & BCC 3463 & DQ341097 & DQ341100 & & Tsui et al. (2007) \\
\hline Boerlagiomyces macrospora & MFLUCC 12-0388 & KU144927 & KU764712 & KU872750 & Doilom et al. (2017) \\
\hline Botryosphaeria dothidea & CBS $115476^{\mathrm{T}}$ & KF766151 & DQ678051 & DQ767637 & Schoch et al. (2006) \\
\hline Chlamydotubeufia aquatica & MFLUCC 16-1131 & KY873625 & KY873620 & KY873284 & Brahamanage et al. (2017) \\
\hline C. chlamydosporum & CBS 160.69 & AY916466 & AY856875 & & Tsui et al. (2006) \\
\hline C. helicospora & MFLUCC 16-0213 & KX454169 & KX454170 & KY117035 & Hyde et al. (2016) \\
\hline C. huaikangplaensis & MFLUCC $10-0926^{\mathrm{T}}$ & JN865210 & JN865198 & & Boonmee et al. (2011) \\
\hline C. khunkornensis & MFLUCC 10-0117 & JN865201 & JN865189 & & Boonmee et al. (2011) \\
\hline C. khunkornensis & MFLUCC 10-0118 & JN865202 & JN865190 & KF301564 & Boonmee et al. (2011) \\
\hline C. krabiensis & MFLUCC 16-1134 & KY678767 & KY678759 & KY792598 & Hyde et al. (2017) \\
\hline Dictyospora thailandica & MFLUCC $16-0001^{\mathrm{T}}$ & KY873627 & KY873622 & KY873286 & Brahamanage et al. (2017) \\
\hline Dictyospora thailandica & MFLUCC 16-0215 & KY873628 & KY873623 & KY873287 & Brahamanage et al. (2017) \\
\hline Helicangiospora lignicola & MFLUCC $11-0378^{\mathrm{T}}$ & KF301523 & KF301531 & KF301552 & Boonmee et al. (2014) \\
\hline Helicoma guttulatum & MFLUCC $16-0022^{\mathrm{T}}$ & KX454171 & KX454172 & MF535254 & Hyde et al. (2016) \\
\hline Helicoma khunkornense & MFLUCC $10-0119^{\mathrm{T}}$ & JN865203 & JN865191 & KF301559 & Boonmee et al. (2011) \\
\hline Helicoma muelleri & CBS 964.69 & AY916453 & AY856877 & & Tsui et al. (2006) \\
\hline Helicomyces roseus & CBS 283.51 & AY916464 & AY856881 & & Tsui and Berbee (2006) \\
\hline Helicomyces talbotii & MUCL 33010 & AY916465 & AY856874 & & Tsui and Berbee (2006) \\
\hline Helicosporium flavum & MFLUCC $16-1230$ & KY873626 & KY873621 & KY873285 & Brahmanage et al. (2017) \\
\hline Helicosporium luteosporum & MFLUCC $16-0226^{\mathrm{T}}$ & KY321324 & KY321327 & KY792601 & Lu et al. (2017a) \\
\hline Helicosporium vegetum & CBS 941.72 & AY916488 & AY856883 & & Tsui et al. (2006) \\
\hline Helicotubeufia guangxiensis & MFLUCC $17-0040^{\mathrm{T}}$ & MH290018 & MH290023 & MH290028 & this study \\
\hline Helicotubeufia guangxiensis & MFLUCC $17-0041$ & МH290019 & MH290024 & MH290029 & this study \\
\hline Helicotubeufia hydei & MFLUCC $17-1980^{\mathrm{T}}$ & MH290021 & MH290026 & MH290031 & this study \\
\hline Helicotubeufia hydei & MFLUCC 17-1986 & MH290022 & MH290027 & MH290032 & this study \\
\hline Helicotubeufia jonesii & MFLUCC $17-0043^{\mathrm{T}}$ & MH290020 & MH290025 & MH290030 & this study \\
\hline Kamalomyces bambusicola & MFLU 11-0228 & & MF506880 & & Phookamsak et al. (2018) \\
\hline Kamalomyces thailandicus & MFLUCC 11-0158 & MF506883 & MF506881 & MF506885 & Phookamsak et al. (2018) \\
\hline Kamalomyces thailandicus & MFLUCC $13-0233^{\mathrm{T}}$ & MF506884 & MF506882 & MF506886 & Phookamsak et al. (2018) \\
\hline Manoharachariella tectonae & MFLUCC $12-0170^{\mathrm{T}}$ & KF301529 & KF301537 & KU872762 & Doilom et al. (2017) \\
\hline Muripulchra aquatica & KUMCC 15-0276 & KY320534 & KY320551 & KY320564 & Luo et al. (2017) \\
\hline Muripulchra aquatica & MFLUCC $15-0249^{\mathrm{T}}$ & KY320532 & KY320549 & & Luo et al. (2017) \\
\hline Neoacanthostigma aquaticum & MFLUCC $17-0049^{\mathrm{T}}$ & KY790444 & KY790432 & KY792608 & Lu et al. (2017b) \\
\hline Neoacanthostigma fusiforme & MFLUCC 11-0510 & KF301529 & KF301537 & & Boonmee et al. (2014) \\
\hline Neohelicomyces aquaticus & MFLUCC $16-0993^{\mathrm{T}}$ & KY320528 & KY320545 & KY320561 & Luo et al. (2017) \\
\hline Neohelicomyces grandisporus & KUMCC $15-0470^{\mathrm{T}}$ & KX454165 & KX454175 & & Luo et al. (2017) \\
\hline Neohelicomyces submersus & MFLUCC $16-1106^{\mathrm{T}}$ & KY320530 & KY320547 & & Luo et al. (2017) \\
\hline Neohelicosporium aquaticum & MFLUCC $17-1519^{\mathrm{T}}$ & MF467916 & MF467929 & MF535242 & Lu et al. (2018) \\
\hline Neohelicosporium parvisporum & MFLUCC $17-1523^{\mathrm{T}}$ & MF467926 & MF467939 & MF535252 & Lu et al. (2018) \\
\hline Neotubeufia krabiensis & MFLUCC $16-1125^{\mathrm{T}}$ & MG012031 & MG012024 & MG012010 & Chaiwan et al. (2017) \\
\hline Tamhinispora indica & NFCCI $2924^{\mathrm{T}}$ & KC469282 & KC469283 & & $\begin{array}{l}\text { Rajeshkumar \& Sharma } \\
\text { (2013) }\end{array}$ \\
\hline Thaxteriellopsis lignicola & MFLUCC 10-0124 & JN865208 & JN865196 & KF301561 & Boonmee et al. (2011) \\
\hline Tubeufia filiformis & MFLUCC $16-1135^{\mathrm{T}}$ & KY092416 & KY092411 & KY117032 & Lu et al. (2017b) \\
\hline Tubeufia javanica & MFLUCC $12-0545^{\mathrm{T}}$ & KJ880034 & KJ880036 & KJ880037 & Boonmee et al. (2014) \\
\hline
\end{tabular}

Notes: Additional sequences (RPB2) for the new taxa in this study are provided as follows (same order as listed in table 1): MH290033, MH290034, MH290036, MH290037 and MH290035

${ }^{\text {a }}$ No data in GenBank.

${ }^{\mathrm{b}}$ Abbreviations of isolates and culture collections: BCC, BIOTEC Culture Collection, Thailand; CBS, Centraalbureau voor Schimmel cultures, Utrecht, The Netherlands; GZCC, Guizhou Culture Collection, Guizhou Academy of Agricultural Sciences, Guiyang, China; KUMCC, Culture Collection of Kunming Institute of Botany, Kunming, China; MFLUCC, Mae Fah Luang University Culture Collection, Chiang Rai, Thailand; MUCL, Mycothèque de l'Université Catholique de Louvain, Louvain-la-Neuve, Belgium; NBRC, the NITE Biological Resource Center; NFCCI, the National Fungal Culture Collection of India. UAMH, UAMH Centre for Global Microfungal Biodiversity, University of Toronto, Canada. T ex-type/ex-epitype isolates. 


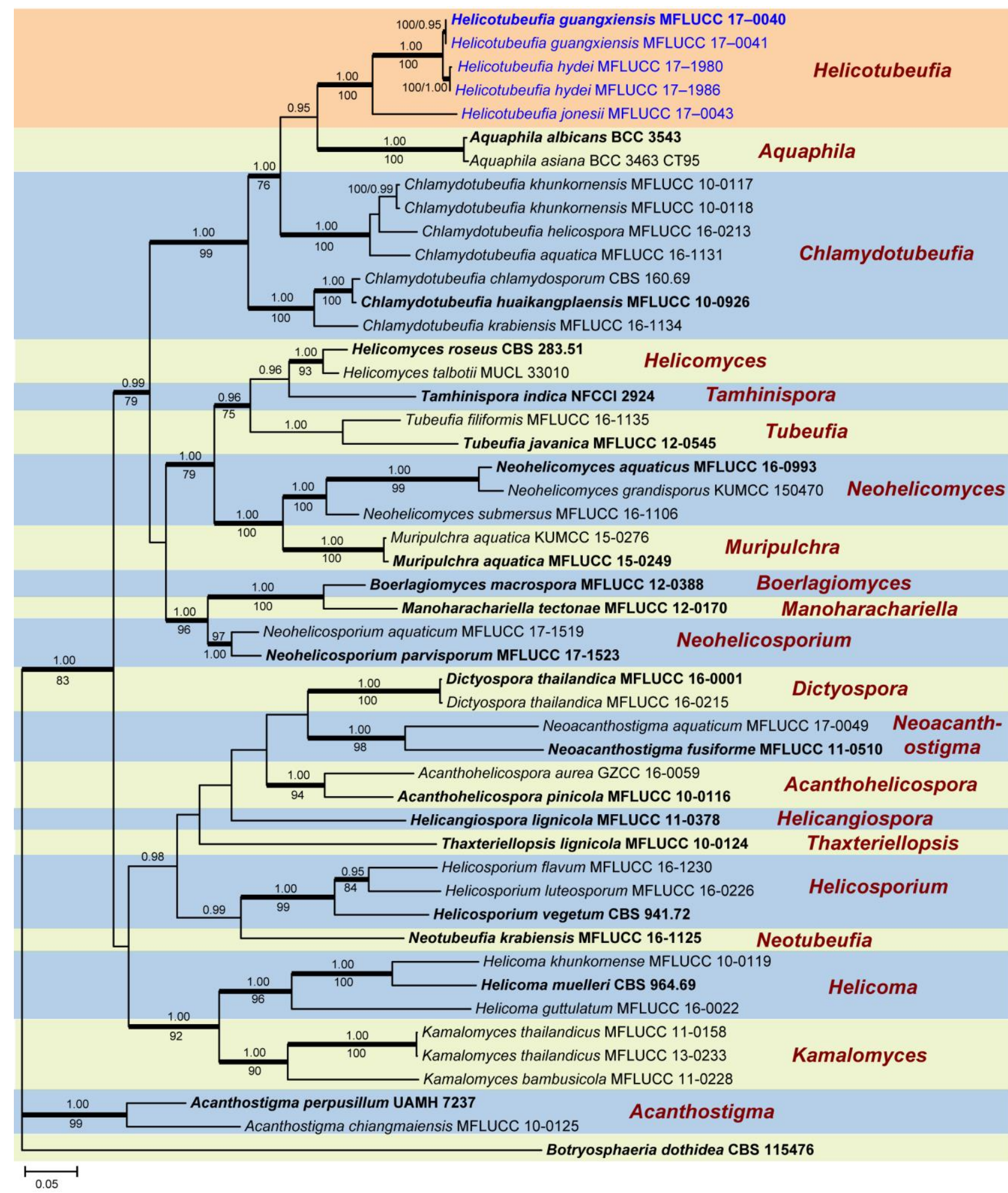

Figure 1 - Consensus phylogram (50 \%) majority rule resulting from a Bayesian analysis of a combined LSU, ITS and TEF1a sequence alignment of Tubeufiaceae. Bayesian posterior probabilities (PP) above 0.95 and ML bootstrap proportion (BP) greater than $75 \%$ are presented at the nodes as BP/PP. Branches with more than $75 \%$ bootstrap (ML/ MP) and 0.95 (PP) are in thickened. The original isolate numbers are noted after the species names, the type species of each genus are in bold, and the new taxa in blue. The scale bar shows 0.05 changes and the tree is rooted to Botryosphaeria dothidea (CBS 115476)

Chlamydotubeufia, and show closer relationship to Aquaphila. Many of the genera in Tubeufiaceae showed the stable phylogenetic positions and relationships, Boerlagiomyces and 
Manoharachariella showed to be a sister group; the genera Helicomyces, Muripulchra, Neohelicomyces and Tubeufia formed a monotypic clade, Helicoma and Kamalomyces formed a sister group, and these clades could be recognized as good genera. However, some of the genera, such as Acanthohelicospora, Acanthostigma, Neoacanthostigma, Neotubeufia and Tamhinispora, their phylogenetic position are unstable. In addition, seven taxa of Chlamydotubeufia formed two distinct monotypic clades, which $C$. chlamydosporum, C. huaikangplaensis (the type) and C. krabiensis clustered together, and $C$. aquatica, C. helicospora and C. khunkornensis formed a separated clade.

\section{Taxonomy}

Helicotubeufia Y.Z. Lu \& J.K. Liu., gen. nov. Index Fungorum number: IF554759; Facesoffungi number: FoF04385

Etymology - "helico" referring to the spiral or helical shape of the conidia and "tubeufia" referring the type genus (Tubeufia) of the Tubeufiaceae.

Saprobic on submerged decaying wood in a freshwater stream. Sexual morph: Ascomata superficial, seated on a subiculum, solitary, scattered, subglobose to ellipsoidal-ovate, dark brown to black, with a central ostiolate. Peridium composed of cells of textura angularis, with inner cells pale brown and outer cells brown. Hamathecium comprising numerous, filiform, septate, branched, hyaline pseudoparaphyses. Asci 8-spored, bitunicate, cylindrical, short or long-pedicellate, apically rounded. Ascospores fusiform, tapering towards rounded ends, slightly curved, guttulate, 5-8septate, not constricted at septa, hyaline, smooth-walled. Asexual morph: hyphomycetous, helicosporous. Conidiophores hyaline, macronematous, partially erect, partially immersed, cylindrical, septate, smooth-walled. Conidiogenous cells holoblastic, polyblastic, sympodial, integrated, terminal or intercalary, cylindrical, with a truncate apex, hyaline, smooth-walled. Conidia acropleurogenous, helicoid, coiled $2 \frac{1}{2}-3 \frac{1}{2}$ times when tightly coiled, becoming loosely coiled in the water, basal cell rounded at tip, multi-septate, not constricted at septa, hyaline, smooth-walled.

Type species - Helicotubeufia guangxiensis Y.Z. Lu \& J.K. Liu

Notes - Helicotubeufia appears to be a typical tubeufiaceous taxon and shares similar morphological characters with species of Tubeufiaceae. Helicotubeufia consists of three species, namely $H$. guangxiensis, $H$. hydei and $H$. jonesii, and formed a well-separated clade from all other genera of Tubeufiaceae. Morphologically, Helicotubeufia is characterized by its superficial, solitary, scattered, subglobose to ellipsoidal-ovate, dark brown to black ascomata; cylindrical asci with hyaline, fusiform, multi-septate, slightly curved and guttulate ascospore and hyphomycetous helicosporous asexual morphs. The new genus is introduced based on multi-gene phylogenetic analysis (Fig. 1).

Helicotubeufia guangxiensis Y.Z. Lu \& J.K. Liu., sp. nov.

Index Fungorum number: IF554760; Facesoffungi number: FoF04386

Figs $2-3$

Etymology - Named referring to the location where the fungus was collected, Guangxi, China.

Holotype - HKAS 97423

Saprobic on submerged decaying wood in a freshwater stream. Sexual morph: Ascomata 220-275 $\mu \mathrm{m}$ high $\times$ 250-290 $\mu \mathrm{m}$ diam., superficial, seated on a subiculum, solitary, scattered, subglobose, dark brown to black, with a central ostiolate. Peridium 50-60 $\mu$ m wide, composed of cells of textura angularis, with inner cells pale brown and outer cells brown. Hamathecium comprising numerous, filiform, septate, branched, hyaline pseudoparaphyses. Asci 120-165 × 13$15.5 \mu \mathrm{m}(\bar{x}=141 \times 14 \mu \mathrm{m}, \mathrm{n}=20), 8$-spored, bitunicate, cylindrical, pedicellate, apically rounded. Ascospores 55-70 $\times 5-6 \mu \mathrm{m}(\bar{x}=60 \times 5.5 \mu \mathrm{m}, \mathrm{n}=50)$, fusiform, tapering towards rounded ends, slightly curved, guttulate, $7-8$-septate, not constricted at septa, hyaline, smooth-walled. 

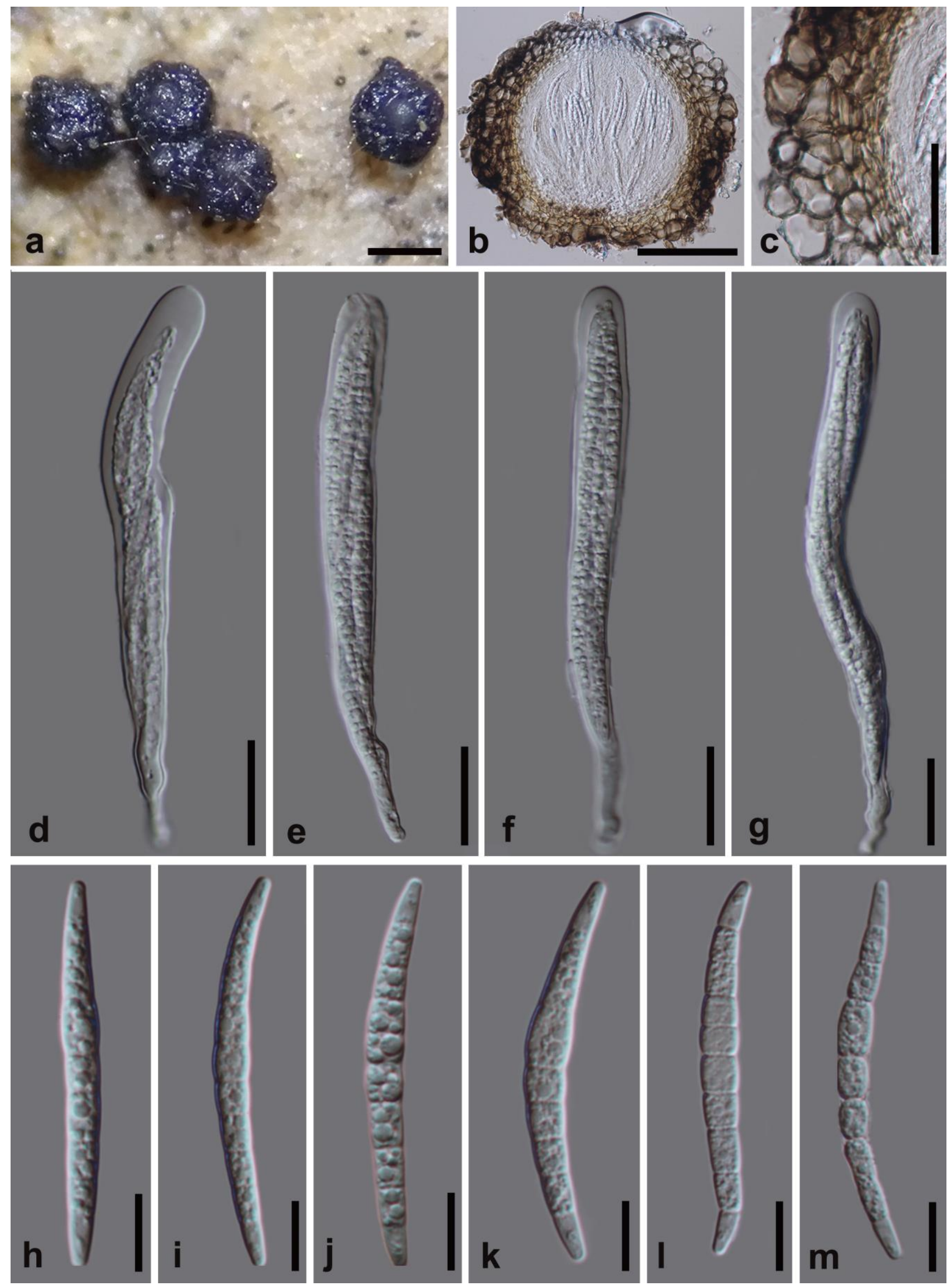

Figure 2 - Helicotubeufia guangxiensis (HKAS 97423, holotype). a Superficial ascomata on substrate. $\mathrm{b}$ Ascoma. c Peridium. d-g Asci. $\mathrm{h}-\mathrm{m}$ Ascospores. Scale bars: $\mathrm{a}=200 \mu \mathrm{m}, \mathrm{b}=100 \mu \mathrm{m}$, $\mathrm{c}=50 \mu \mathrm{m}, \mathrm{d}-\mathrm{g}=20 \mu \mathrm{m}, \mathrm{h}-\mathrm{m}=10 \mu \mathrm{m}$ 

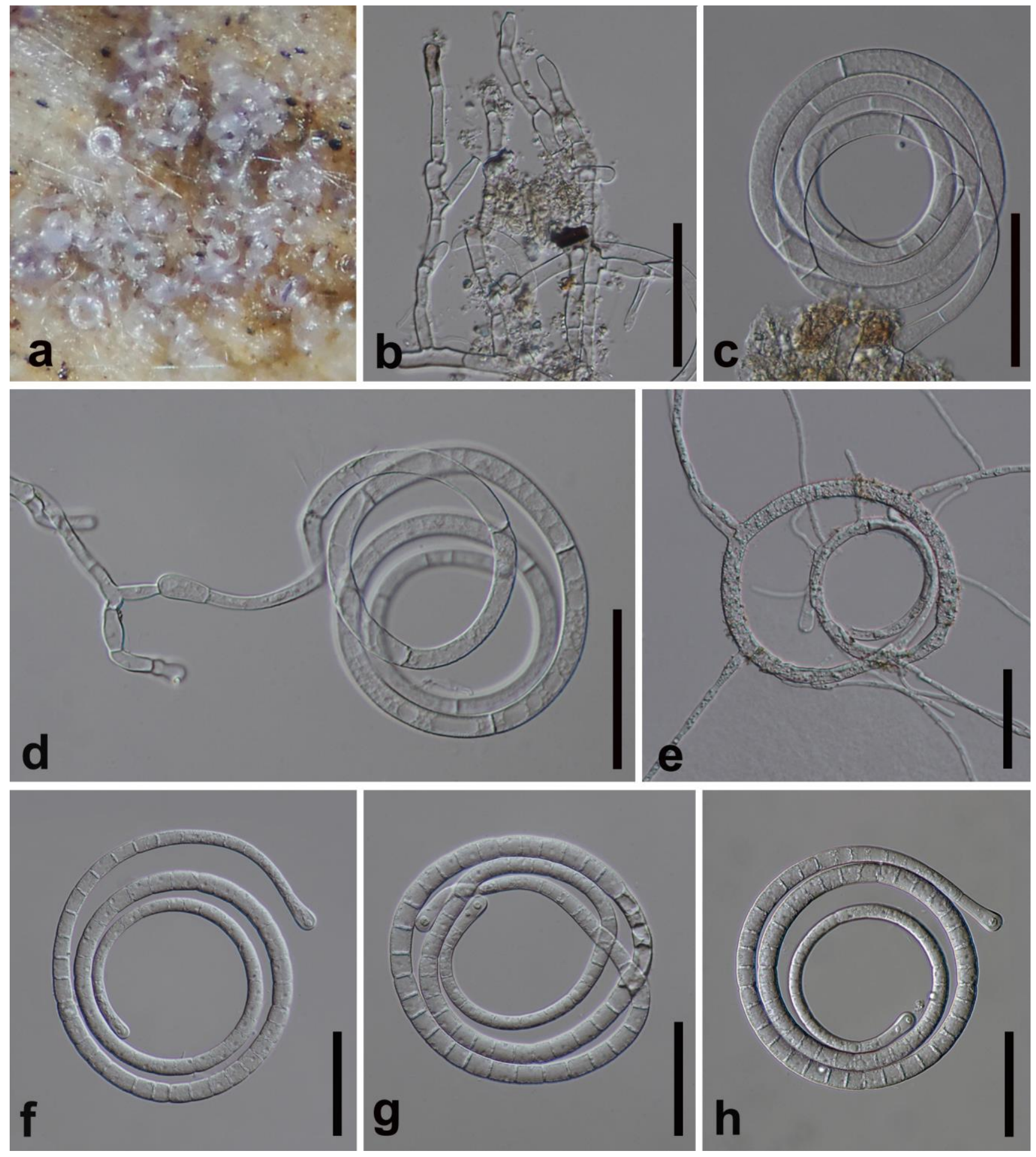

Figure 3 - Helicotubeufia guangxiensis (HKAS 97424, paratype). a Colony on decaying wood. b Conidiophores. $\mathrm{c}-\mathrm{d}$ Conidiophores with attached conidia. e Germinating conidium $\mathrm{f}-\mathrm{h}$ Conidia. Scale bars: $\mathrm{b}-\mathrm{h}=50 \mu \mathrm{m}$.

Asexual morph: hyphomycetous, helicosporous. Conidiophores macronematous, partially erect, partially immersed, cylindrical, septate, 70-140 $\mu \mathrm{m}$ long, 4-6 $\mu \mathrm{m}$ wide, hyaline, smooth-walled. Conidiogenous cells holoblastic, monoblastic, sympodial, integrated, terminal or intercalary, cylindrical, with a truncate apex, 10-18 $\mu \mathrm{m}$ long, 4-6 $\mu \mathrm{m}$ wide, hyaline, smooth-walled. Conidia acropleurogenous, helicoid, basal cell rounded at tip, 85-120 $\mu \mathrm{m}$ diam. and conidial filament 7-10 $\mu \mathrm{m}$ wide $(\bar{x}=105 \times 9 \mu \mathrm{m}, \mathrm{n}=20), 590-845 \mu \mathrm{m}$ long, coiled $2 \frac{1}{2}-3 \frac{1}{2} 2$ times when tightly coiled, becoming loosely coiled in the water, multi-septate, up to 83-septate, not constricted at septa, hyaline, smooth-walled. 
Culture characteristics - Conidia germinating on water agar (WA) within $24 \mathrm{~h}$ and germ tubes produced from conidia. Colonies growing on PDA, circular, with center umbonate surface, edge entire, reaching $11 \mathrm{~mm}$ in 3 weeks at $28{ }^{\circ} \mathrm{C}$, pale brown to brown in PDA medium. mycelium superficial and partially immersed, branched, septate, hyaline to pale brown, smooth.

Material examined - CHINA, Guangxi Province, Fang Cheng Gang City, on submerged decaying wood in a freshwater stream, 15 May 2016, Yong-Zhong Lu, JHC04-1 (HKAS 97423, holotype; GZAAS 16-0045, isotype), ex-type living culture, MFLUCC 17-0040 = GZCC 160033; Ibid., JHC04-2 (HKAS 97424 = GZAAS 16-0106, paratype), living culture, MFLUCC 17$0041=$ GZCC 16-0094.

Notes - Helicotubeufia guangxiensis is designated as the type species of Helicotubeufia with both asexual and sexual morphs found from the substrates in nature. Morphologically, the sexual morph of H. guangxiensis resembles to Neotubeufia krabiensis in having subglobose ascomata with a central ostiolate, thick peridium, cylindrical bitunicate asci and fusiform ascospores, but can be

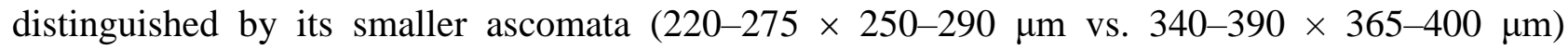
(Chaiwan et al. 2017). The asexual morph of $H$. guangxiensis differs from other helicosporous genera by its mycelium and conidiophores with a large sized conidia are hyaline, while most of other helicosporous hyphomycetes are mycelium and conidiophores pale brown to brown, or hyaline conidiophores with a small sized conidia (Linder 1929, Moore 1957, Goos 1985, 1986, 1989, Zhao et al. 2007, Boonmee et al. 2014, Lu et al. 2017c, Luo et al. 2017). In addition, all of previously described helicosporous species which conidial filament is wider than $7 \mu \mathrm{m}$ and have pale brown to brown conidia, but the conidia of our new isolates are hyaline (Goos 1989, Lu et al. 2017c).

Helicotubeufia hydei Y.Z. Lu \& J.K. Liu., sp. nov.

Fig. 4

Index Fungorum number: IF554761; Facesoffungi number: FoF04387

Etymology: Named in honour of Kevin D. Hyde for his contributions to Asian mycology.

Holotype: MFLU 17-1122

Saprobic on decaying wood in a dry freshwater stream. Sexual morph: Ascomata 285-350 $\mu \mathrm{m}$ high $\times 270-375 \mu \mathrm{m}$ diam., superficial, seated on a subiculum, solitary, scattered, subglobose, dark brown to black, with a central ostiolate. Peridium 80-105 $\mu \mathrm{m}$ wide, composed of cells of textura angularis, with inner cells pale brown and outer cells brown. Hamathecium 1-2 $\mu \mathrm{m}$ wide, comprising numerous, filiform, septate, branched, hyaline pseudoparaphyses. Asci $90-110 \times 13-16$ $\mu \mathrm{m}(\bar{x}=100 \times 14.5 \mu \mathrm{m}, \mathrm{n}=10), 8$-spored, bitunicate, cylindrical, short-pedicellate, apically rounded. Ascospores 45-65 $\times 4.5-6.5 \mu \mathrm{m}(\bar{x}=55 \times 5.5 \mu \mathrm{m}, \mathrm{n}=50)$, fusiform, tapering towards rounded ends, slightly curved, guttulate, 5-8-septate, not constricted at septa, hyaline, smoothwalled. Asexual morph: undetermined.

Culture characteristics - Ascospores germinating on water agar (WA) within $36 \mathrm{~h}$ and germ tubes produced from ascospores. Colonies growing on PDA, circular, with flat surface, edge entire, reaching $10 \mathrm{~mm}$ in 2 weeks at $28{ }^{\circ} \mathrm{C}$, pale brown to brown in PDA medium. Mycelium superficial and partially immersed, branched, septate, hyaline to pale brown, smooth.

Material examined - THAILAND, Trat, Amphoe Ko Chang, Yuttha Navi Ko Chang Memorial, on decaying wood in a dry stream, 27 April 2017, Yong-Zhong Lu, TD05 (MFLU 171122, holotype; HKAS100792, isotype); ex-type living culture, MFLUCC 17-1980; Ibid., TD12 (HKAS 100798, paratype); living culture, MFLUCC 17-1986.

Notes - Morphologically, Helicotubeufia hydei resembles to H. guangxiensis, but can differ by its larger ascomata $(285-350 \times 270-375 \mu \mathrm{m}$ vs. $220-275 \times 250-290 \mu \mathrm{m})$ and thicker peridium $(80-105 \mu \mathrm{m}$ vs. $50-60 \mu \mathrm{m})$, as well as the shorter asci $(90-110 \mu \mathrm{m}$ vs. $120-165 \mu \mathrm{m})$. Phylogenetically, they are distinct species (Fig. 1).

Helicotubeufia jonesii Y.Z. Lu \& J.K. Liu., sp. nov. Fig. 5 Index Fungorum number: IF554762; Facesoffungi number: FoF04388 
Etymology - Named in honour of Prof. E.B. Gareth Jones for his contributions to Asian mycology.

Holotype - HKAS 97426

Saprobic on submerged decaying wood in a freshwater stream. Sexual morph: Ascomata 170-215 $\mu \mathrm{m}$ high $\times 175-215 \mu \mathrm{m}$ diam., superficial, seated on a subiculum, solitary, scattered, subglobose, ellipsoidal-ovate, dark brown to black, with a central ostiolate. Peridium 35-45 $\mu \mathrm{m}$ wide, composed of cells of textura angularis, with inner cells brown and outer cells dark brown. Hamathecium comprising numerous, filiform, septate, branched, hyaline pseudoparaphyses. Asci 95-120 × 15-17.5 $\mathrm{m}(\bar{x}=105 \times 16 \mu \mathrm{m}, \mathrm{n}=20)$, 8-spored, bitunicate, cylindrical, shortpedicellate, apically rounded. Ascospores 35-43 $(-47) \times 4.5-7 \mu \mathrm{m}(\bar{x}=40 \times 5.5 \mu \mathrm{m}, \mathrm{n}=50)$, fusiform, tapering towards rounded ends, enlarged at the $3^{\text {rd }}$ cell, slightly curved, guttulate, 5-6septate, not constricted at septa, hyaline, smooth-walled. Asexual morph: undetermined.

Culture characteristics - Ascospores germinating on water agar (WA) within $12 \mathrm{~h}$ and germ tubes produced from ascospores. Colonies growing on PDA, circular, with flat surface, edge entire, reaching $14 \mathrm{~mm}$ in 3 weeks at $28{ }^{\circ} \mathrm{C}$, brown to dark brown in PDA medium. Mycelium superficial and partially immersed, branched, septate, hyaline to pale brown, smooth.

Material examined - CHINA, Guangxi Province, Fang Cheng Gang City, on submerged decaying wood in a freshwater stream, 15 May 2016, Yong-Zhong Lu, JHC08-2 (HKAS 97426, holotype; GZAAS 16-0052, isotype), ex-type living culture, MFLUCC 17-0043, GZCC 16-0040.

Notes - Helicotubeufia jonesii morphologically resembles to H. guangxiensis and $H$. hydei in ascomata and asci, but can be easily distinguished by its ascospores which are obviously enlarged at the $3^{\text {rd }}$ cell, while the other two species lack this characteristic. In addition, the ascomata of $H$. jonesii are smaller and its peridium is thinner than those of $H$. guangxiensis and $H$. hydei. The phylogenetic analysis of combined LSU, ITS and TEF1 $\alpha$ sequences data showed that they are phylogenetically distinct species, and $H$. guangxiensis and $H$. hydei have a close phylogenetic relationship.

\section{Discussion}

The asexual ascomycetes comprise a large number of genera that have never been linked to a sexual morph (Shenoy et al. 2007). Helicosporous hyphomycete genera are commonly found in Tubeufiaceae, and several genera have been recognized. Many share a similar morphology and could be morphologically assigned as a group, but they are phylogenetically distinct (Boonmee et al. 2011, 2014, Lu et al. 2017a, c, 2018, Luo et al. 2017). This is not only at the species level, but even genus ( $\mathrm{Lu}$ et al. 2017a, 2018, Luo et al. 2017). Lu et al. (2018) suggested that many helicosporous species may be wrongly named, even at the genus level; and this can be confirmed by future studies which bring more molecular data into the study. With more and more molecular data of the Tubeufiales become available, the understanding of the taxonomy and phylogeny of the order have been well investigated (Kodsueb et al. 2006, Boonmee et al. 2011, 2014, Liu et al. 2015, Brahmanage et al. 2017, Doilom et al. 2017, Lu et al. 2017a, b, Luo et al. 2017). Boonmee et al. (2014) provided a modern classification of the order Tubeufiales based on the examinations of the types and phylogenetic analysis of multi-gene molecular data, including the asexual and sexual morphs. Subsequently, several new taxa were introduced mainly based on the phylogenetic evidences (Hyde et al. 2016, Brahmanage et al. 2017, Chaiwan et al. 2017, Lu et al. 2017b, c, 2018, Luo et al. 2017) which the morphological characters are similar.

One interesting finding in this study is that the phylogenetic position of Chlamydotubeufia is questionable. In previous studies (Brahmanage et al 2017, Chaiwan et al 2017, Lu et al 2017a, b, 2018, Luo et al 2017, Phookamsak et al. 2018), Chlamydotubeufia and Aquaphila have been shown to be a stable sister group, and close to Boerlagiomyces and Manoharachariella. However, in this study, where five taxa of Helicotubeufia were added to the phylogenetic analysis of Tubeufiaceae, the seven isolates representing six species of Chlamydotubeufia formed two clades, where $C$. chlamydosporum, $C$. huaikangplaensis (the type) and C. krabiensis clustered together, and $C$. aquatica, C. helicospora and C. khunkornensis formed a separated clade. 

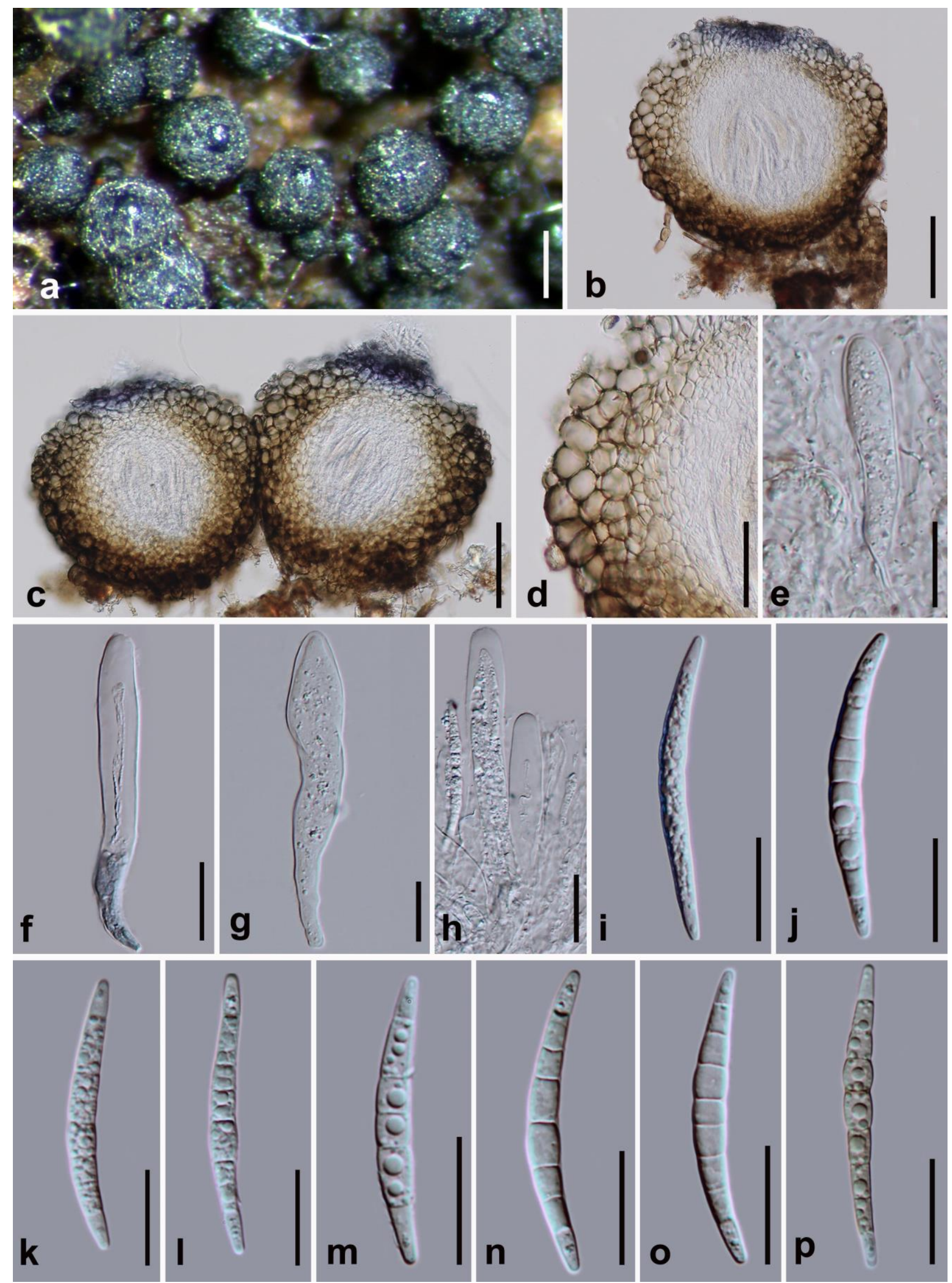

Figure 4 - Helicotubeufia hydei (MFLU 17-1122, holotype). a Superficial ascomata on substrate. b-c Ascoma. d Peridium. e-h Asci. i-p Ascospores. Scale bars: $\mathrm{a}=200 \mu \mathrm{m}, \mathrm{b}-\mathrm{c}=100 \mu \mathrm{m}$, $\mathrm{d}=50 \mu m, \mathrm{e}-\mathrm{p}=20 \mu \mathrm{m}$. 

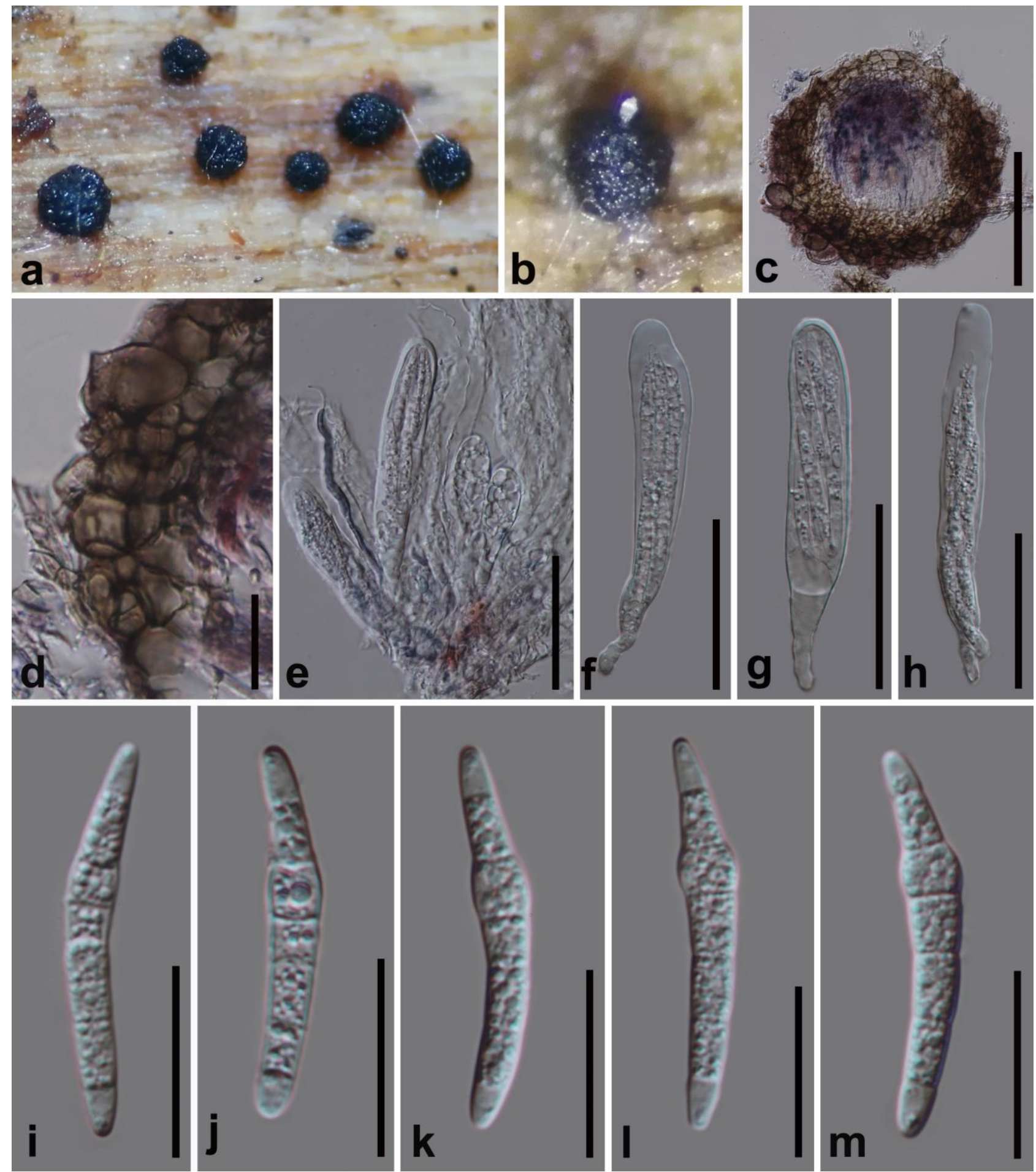

Figure 5 - Helicotubeufia jonesii (HKAS 97426, holotype). a-b Superficial ascomata on substrate. c Ascoma. d Peridium. e- $\mathrm{h}$ Asci. i-q Ascospores. $\mathrm{r}-\mathrm{s}$ Colonies on PDA from above and below. Scale bars: $\mathrm{c}=100 \mu \mathrm{m}, \mathrm{d}=20 \mu \mathrm{m}, \mathrm{e}-\mathrm{h}=50 \mu \mathrm{m}, \mathrm{i}-\mathrm{m}=20 \mu \mathrm{m}$.

Based on both morphology and phylogeny, we can treat $C$. aquatica, $C$. helicospora and $C$. khunkornensis as Chlamydotubeufia sensu lato and C. chlamydosporum, C. huaikangplaensis and $C$. krabiensis as Chlamydotubeufia sensu stricto. This also indicates that $C$. aquatica, $C$. helicospora and $C$. khunkornensis could be recognized as a new genus and the monotypic clade including $C$. chlamydosporum, $C$. krabiensis and the type species $C$. huaikangplaensis could represent the genus Chlamydotubeufia. However, we are not willing to follow this conclusion until more taxa and sequences data are available, as well as more sampling and taxa population included 
in the analysis. This funding also confirms that the tubeufiaceous taxa support a high diversity and the phylogenetic studies give a better understanding towards the classification of both asexual and sexual morphs of Tubeufiales.

\section{Acknowledgements}

This work was supported by funding from the National Natural Science Foundation of China (NSFC 31600032) and Science and Technology Foundation of Guizhou Province (LH [2015]7061). Jian-Kui Liu thanks Chiang Mai University (Chiang Mai, Thailand) for a Post-Doctoral Fellowship and Mae Fah Luang University for the offer of a short term Visiting Professorship in 2017.

\section{References}

Boonmee S, Rossman AY, Liu JK, Li WJ et al. 2014 - Tubeufiales, ord. nov., integrating sexual and asexual generic names. Fungal Diversity 68, 239-298.

Boonmee S, Zhang Y, Chomnunti P, Chukeatirote E et al. 2011 - Revision of lignicolous Tubeufiaceae based on morphological reexamination and phylogenetic analysis. Fungal Diversity 51, 63-102.

Brahmanage RS, Lu YZ, Bhat DJ, Wanasinghe DN et al. 2017 - Phylogenetic investigations on freshwater fungi in Tubeufiaceae (Tubeufiales) reveals the new genus Dictyospora and new species Chlamydotubeufia aquatica and Helicosporium flavum. Mycosphere 8, 917-933.

Cai L, Tsui CKM, Zhang KQ, Hyde KD. 2002 - Aquatic fungi from Lake Fuxian, Yunnan, China. Fungal Diversity 9, 57-70.

Chaiwan N, Lu YZ, Tibpromma S, Bhat DJ et al. 2017 - Neotubeufia gen. nov. and Tubeufia guangxiensis sp. nov. (Tubeufiaceae) from freshwater habitats. Mycosphere 8, 1443-1456.

Doilom M, Dissanayake AJ, Wanasinghe DN, Boonmee S et al. 2017 - Microfungi on Tectona grandis (teak) in Northern Thailand. Fungal Diversity 82, 107-182.

Goos RD. 1985 - A review of the anamorph genus Helicomyces. Mycologia 77, 606-618.

Goos RD. 1986 - A review of the anamorph genus Helicoma. Mycologia 78, 744-761.

Goos RD. 1989 - On the anamorph genera Helicosporium and Drepanospora. Mycologia 81, 356374.

Hall TA. 1999 - BioEdit: a user-friendly biological sequence alignment editor and analysis program for Windows 95/98/NT. Nucleic Acids Symposium Series 41, 95-98.

Hillis DM, Bull JJ. 1993 - An empirical test of bootstrapping as a method for assessing confidence in phylogenetic analysis. Systematic Biology 42, 182.

Ho WH, Hyde KD, Hodgkiss IJ, Yanna 2001 - Fungal communities on submerged wood from streams in Brunei, Hong Kong, and Malaysia. Mycological Research 105, 1492-1501.

Huelsenbeck JP, Ronquist F. 2001 - MRBAYES: Bayesian inference of phylogenetic trees. Bioinformatics 17: 754-755. https://doi.org/10.1093/bioinformatics/17.8.754

Hyde KD, Goh TK 1998 - Fungi on submerged wood in Lake Barrine, north Queensland, Australia. Mycological Research 102, 739-749.

Hyde KD, Jones EBG, Liu JK, Ariyawansa H et al. 2013 - Families of Dothideomycetes. Fungal Diversity 63, 1-313.

Hyde KD, Fryar S, Tian Q, Bahkali AH, Xu JC. 2016 - Lignicolous freshwater fungi along a northsouth latitudinal gradient in the Asian/Australian region; can we predict the impact of global warming on biodiversity and function? Fungal Ecology 19, 190-200.

Index Fungorum 2018 - Available from: http://www.indexfungorum.org/Names/Names.asp (accessed April 2018)

Jayasiri SC, Hyde KD, Ariyawansa HA, Bhat J et al. 2015 - The Faces of Fungi database: fungal names linked with morphology, phylogeny and human impacts. Fungal Diversity 74, 3-18.

Kodsueb R, Jeewon R, Vijaykrishna D, McKenzie EHC et al. 2006 - Systematic revision of Tubeufiaceae based on morphological and molecular data. Fungal Diversity 21, 105-130.

Linder DH. 1929 - A monograph of the helicosporous fungi imperfecti. Annals of the Missouri 
Botanical Garden 16, 227-388.

Liu JK, Chomnunti P, Cai L, Phookamsak R et al. 2010 - Phylogeny and morphology of Neodeightonia palmicola sp. nov. from palms. Sydowia 62, 261-276.

Liu JK, Hyde KD, Jones EG, Ariyawansa HA et al. 2015 - Fungal diversity notes 1-110: taxonomic and phylogenetic contributions to fungal species. Fungal Diversity 72, 1-197.

Liu JK, Hyde KD, Jeewon R, Phillips et al. 2017 - Ranking higher taxa using divergence times: a case study in Dothideomycetes. Fungal Diversity 84, 75-99.

Lu YZ, Boonmee S, Bhat DJ, Hyde KD, Kang JC. 2017a - Helicosporium luteosporum sp. nov. and Acanthohelicospora aurea (Tubeufiaceae, Tubeufiales) from terrestrial habitats. Phytotaxa 319, 241-253.

Lu YZ, Boonmee S, Dai DQ, Liu JK et al. 2017b - Four new species of Tubeufia (Tubeufiaceae, Tubeufiales) from Thailand. Mycological Progress 16, 403-417.

Lu YZ, Boonmee S, Liu JK, Hyde KD et al. 2017c - Novel Neoacanthostigma species from aquatic habitats. Cryptogamie Mycologie 38, 169-190.

Lu YZ, Boonmee S, Liu JK, Hyde KD et al. 2018 - Multi-gene phylogenetic analyses reveals Neohelicosporium gen. nov. and five new species of helicosporous hyphomycetes from aquatic habitats. Mycological Progress 17, 631-646.

Luo ZL, Bhat DJ, Jeewon R, Boonmee S et al. 2017 - Molecular phylogeny and morphological characterization of asexual fungi (Tubeufiaceae) from freshwater habitats in Yunnan, China. Cryptogamie Mycologie 38, 1-28.

Maharachchikumbura SSN, Hyde KD, Jones EG, McKenzie EH et al. 2015 - Towards a natural classification and backbone tree for Sordariomycetes. Fungal Diversity 72, 199-301.

Miller MA, Pfeiffer W, Schwartz T. 2010 - Creating the CIPRES science gateway for inference of large phylogenetic trees. Proceedings of the Gateway Computing Environments Workshop (GCE), November 14, 2010, New Orleans. Louisiana, pp. 1-8. https://doi.org/10.1109/GCE.2010.5676129

Moore RT. 1957 - Index to the Helicosporae: addenda. Mycologia 49, 580-587.

Nylander JAA. 2004 - MrModeltest 2.0. Program distributed by the author. Evolutionary Biology Centre, Uppsala University.

Page RDM. 1996 - TreeView: an application to display phylogenetic trees on personal computers. Computer applications in the biosciences 12, 357-358.

Phookamsak R, Lu YZ, Hyde KD, Jeewon R et al. 2018 - Phylogenetic characterization of two novel Kamalomyces species in Tubeufiaceae (Tubeufiales). Mycological Progress 17, 647660.

Pinnoi A, Lumyong S, Hyde KD, Jones EBG. 2006 - Biodiversity of fungi on the palm Eleiodoxa conferta in Sirindhorn peat swamp forest, Narathiwat, Thailand. Fungal Diversity 22, 205218.

Pinruan U, Hyde KD, Lumyong S, McKenzie EHC, Jones EBG 2007 - Occurrence of fungi on tissues of the peat swamp palm Licuala longicalycata. Fungal Diversity 25, 157-173.

Promputtha I, Miller AN. 2010 - Three new species of Acanthostigma (Tubeufiaceae, Dothideomycetes) from Great Smoky Mountains National Park. Mycologia 102, 574-587.

Rajeshkumar KC, Sharma R. 2013 - Tamhinispora a new genus belongs to family Tubeufiaceae from the Western Ghats, India based on morphology and phylogenetic analysis. Mycosphere $4(2), 165-175$.

Rambaut A, Suchard M, Drummond AJ 2013 - Tracer 1.6. Available from http://tree.bio.ed.ac.uk/software/tracer/ (accessed 1 April 2018)

Rannala B, Yang Z. 1996 - Probability distribution of molecular evolutionary trees: a new method of phylogenetic inference. Journal of Molecular Evolution 43, 304-311.

Rehner SA, Buckley E. 2005 - A Beauveria phylogeny inferred from nuclear ITS and EF1- $\alpha$ sequence: evidence for cryptic diversification and links to Cordyceps teleomorphs. Mycologia 97, 84-98. 
Shearer CA, Raja HA, Miller AN, Nelson P et al. 2009 - The molecular phylogeny of freshwater Dothideomycetes. Studies in Mycology 64, 145-153.

Shenoy BD, Jeewon R, Hyde KD. 2007 - Impact of DNA sequence-data on the taxonomy of anamorphic fungi. Fungal Diversity 26, 1-54.

Stamatakis A. 2006 - RAxML-VI-HPC: maximum likelihood-based phylogenetic analyses with thousands of taxa and mixed models. Bioinformatics 22, 2688-2690. http://dx.doi.org/10.1093/bioinformatics/bt1446

Swofford DL. 2002 - PAUP: phylogenetic analysis using parsimony, version 4.0 b10. Sunderland, MA: Sinauer Associates.

Tamura K, Peterson D, Peterson N, Stecher G et al. 2011 - MEGA5: Molecular Evolutionary Genetics Analysis using Maximum Likelihood, Evolutionary Distance, and Maximum Parsimony Methods. Molecular Biology and Evolution 28, 2731-2739.

Thompson JD, Gibson TJ, Plewniak F, Jeanmougin F, Higgins DG. 1997 - The CLUSTAL_X windows interface: flexible strategies for multiple sequence alignment aided by quality analysis tools. Nucleic Acids Research 25, 4876-4882.

Tsui CKM, Berbee ML. 2006 - Phylogenetic relationships and convergence of helicosporous fungi inferred from ribosomal DNA sequences. Molecular Phylogenetics and Evolution 39, 587597.

Tsui CKM, Sivichai S, Berbee ML. 2006 - Molecular systematics of Helicoma, Helicomyces and Helicosporium and their teleomorphs inferred from rDNA sequences. Mycologia 98, 94-104.

Tsui CKM, Sivichai S, Rossman AY, Berbee ML. 2007 - Tubeufia asiana, the teleomorph of Aquaphila albicans in the Tubeufiaceae, Pleosporales, based on cultural and molecular data. Mycologia 99, 884-894.

Vijaykrishna D, Jeewon R, Hyde KD. 2006 - Molecular taxonomy, origins and evolution of freshwater ascomycetes. Fungal Diversity 23, 351-390.

Vilgalys R, Hester M. 1990 - Rapid genetic identification and mapping of enzymatically amplified ribosomal DNA from several Cryptococcus species. Journal of Bacteriology 172, 4238-4246.

White TJ, Bruns TD, Lee SB, Taylor JW. 1990 - Amplification and direct sequencing of fungal ribosomal RNA genes for phylogenetics. In: Innis MA, Gelfand DH, Sninsky JJ, White TJ (eds) PCR protocols: a guide to methods and applications. Academic Press, San Diego, California, 315-322.

Wijayawardene NN, Hyde KD, Rajeshkumar KC, Hawksworth DL et al. 2017 - Notes for genera: Ascomycota. Fungal Diversity 86, 1-594.

Wijayawardene NN, Hyde KD, Lumbsch HT, Liu JK et al. 2018 - Outline of Ascomycota: 2017. Fungal Diversity 88, 167-263.

Wong MK, Goh TK, Hodgkiss IJ, Hyde KD et al. 1998 - Role of fungi in freshwater ecosystems. Biodiversity \& Conservation 7, 1187-1206.

Zhao GZ, Liu X, Wu W. 2007 - Helicosporous hyphomycetes from China. Fungal Diversity 26, 313-524.

Zhaxybayeva O, Gogarten JP. 2002 - Bootstrap, Bayesian probability and maximum likelihood mapping: exploring new tools for compa 